\title{
ITERATES OF MEASURABLE TRANSFORMATIONS AND MARKOV OPERATORS
}

\author{
BY \\ ARSHAG B. HAJIAN AND YUJI ITO
}

1. Introduction. The existence of a finite, invariant, and equivalent measure for a measurable transformation $\phi$ and for a Markov process $P(x, B)$ has been investigated in detail by many authors. In [1] and [4] the reader may find most of the necessary and sufficient conditions for the problem. Some of these conditions furnish useful information to other related problems and help in understanding the nature of measurable transformations and Markov processes in general.

In this paper we discuss two new conditions on a measurable transformation in terms of its iterates; we also consider their generalizations to Markov processes and the operators induced by them.

The first condition, see (1) below, states that the infinite sum $\sum_{i=1}^{\infty} f\left(\phi^{n_{i}} x\right)$ diverges almost everywhere for every measurable function $f(x)$ with $f(x)>0$ a.e., and for every sequence of integers $\left\{n_{i} \mid i=1,2, \cdots\right\}$. This condition may be regarded as a generalization of a known condition on a measurable transformation used in proving general ergodic theorems; namely, the infinite sum $\sum_{n=1}^{\infty} f\left(\phi^{n} x\right)$ diverges almost everywhere for every measurable function $f(x)$ with $f(x)>0$ a.e. For a one-to-one measurable transformation $\phi$ it is easy to see that this last condition is equivalent to the nonexistence of a wandering set of positive measure. In this paper we show (Theorem 1) that condition (1) is equivalent to the nonexistence of a weakly wandering set of positive measure.

The second condition we consider, see (4) below, states that for any decomposition $\left\{A_{k} \mid k=1,2, \cdots\right\}$ of the space $X$ into mutually disjoint measurable sets, for any sequence of integers $\left\{n_{i} \mid i=1,2, \cdots\right\}$, and for almost all $x \in X$ there exists some $k=k(x)$ such that $\phi^{n_{i}} x \in A_{k}$ for infinitely many $i$. We show (Theorem 2) that condition (4) is equivalent to condition(1). The notion of a weakly wandering set was introduced in [1] and used in studying the problem of invariant measures. It follows (corollary to Theorem 2) as a direct consequence of Theorem 1 of [1] that conditions (1) and (4) are again equivalent conditions to the existence of a finite, invariant, and equivalent measure for a given measurable and nonsingular transformation. As a direct consequence of the above corollary it follows that a measurable transformation satisfying conditions (1) or (4) is necessarily recurrent; this means that for every measurable set $B$ of positive measure and for almost all

Received by the editors October 16, 1963. 
$x \in B$ there existinfinitely many $n$ such that $\phi^{n} x \in B$. Since recurrent transformations exist which do not admit a finite, invariant, and equivalent measure (namely, ergodic measure-preserving transformations defined on an infinite measure space), it is clear that the converse implication is not true in general.

In $\S 3$ we study transformations of the above type in more detail. Using the notion of a weakly wandering sequence, we show (Theorem 3 and Corollary 2 of Theorem 3) that conditions (1) and (4) are violated in a stronger way for ergodic measure-preserving transformations defined on an infinite measure space.

In $\S 4$ we study the analogue of the above conditions for a Markov process and for the operators induced by it. We note that the above conditions when applied to a Markov process or to the operators induced by it have to be modified somewhat in order to be applicable, see conditions (12), (14), and (17). Furthermore, it turns out (Theorems 4, 5, and 6) that the analogous conditions, when applied to the respective operators, give somewhat different results than in the case of a measurable transformation. It is also interesting to note that in $\$ 2$ we require the transformation $\phi$ to be one-to-one; the example given in the proof of Theorem 5 shows this significance.

2. Measurable transformations. We let $(X, \mathscr{B})$ be a measurable space, where $X=\{x\}$ is an abstract set of points and $\mathscr{B}=\{B\}$ is a $\sigma$-field of subsets of $X$ with $X \in \mathscr{B}$. By a measure $m$ defined on $\mathscr{B}$ we mean a non-negative, real-valued and countably additive set function with the property that the measure of the whole space $X$ is $\sigma$-finite. We say that a measurable set $B$ is a null set if $m(B)=0$. Two measures $m$ and $m^{\prime}$ defined on the same measurable space $(X, \mathscr{B})$ are said to be equivalent if they have the same null sets. Unless otherwise specified, we shall consider a one-to-one transformation $\phi$ of $X$ onto itself; we assume that the transformation $\phi$ is measurable, i.e., a set $B \in \mathscr{B}$ if and only if $\phi B \in \mathscr{B}$, and that $\phi$ is nonsingular, i.e., $m(B)=0$ if and only if $m(\phi B)=0$ for every $B \in \mathscr{B}$.

If for a measurable set $W$ there exists a sequence of integres $\left\{n_{i} \mid i=1,2, \cdots\right\}$ such that

$$
\phi^{n_{i}} W \cap \phi^{n_{j}} W=\varnothing \quad \text { for } i \neq j,
$$

we say that $W$ is a weakly wandering set under the sequence $\left\{n_{i} \mid i=1,2, \cdots\right\}$; in case $n_{i}=i$ for $i=1,2, \cdots$ we say that $W$ is a wandering set. By the characteristic function $\chi_{B}(x)$ of a measurable set $B$ we mean the real-valued function defined on $X$ as follows:

$$
\chi_{B}(x)= \begin{cases}1 & \text { if } x \in B \\ 0 & \text { if } x \in X-B .\end{cases}
$$

THEOREM 1. Let $\phi$ be a measurable and nonsingular transformation defined on a $\sigma$-finite measure space $(X, \mathscr{B}, m)$. Then there does not exist any weakly wandering set of positive measure if and only if 
for any sequence of integers $\left\{n_{i} \mid i=1,2, \cdots\right\}$ and any measurable function $f(x)$ with $f(x)>0$ a.e. we have

$$
\sum_{i=1}^{\infty} f\left(\phi^{n_{i}} x\right)=\infty \text { a.e. }
$$

Proof. Let $W$ be a set of positive measure and weakly wandering under the sequence $\left\{n_{i} \mid i=1,2, \cdots\right\}$. We define the real-valued measurable function $f(x)$ on $X$ as follows:

$$
f(x)=\left\{\begin{array}{cl}
\frac{1}{2_{i}} & \text { if } x \in \phi^{n_{i}} W \text { for } i=1,2, \cdots, \\
1 & \text { if } x \notin \bigcup_{i=1}^{\infty} \phi^{n_{i}} W .
\end{array}\right.
$$

It follows then that for the above sequence $\left\{n_{i}\right\}$ we have

$$
\sum_{i=1}^{\infty} f\left(\phi^{n_{i}} x\right)=\sum_{i=1}^{\infty} \frac{1}{2^{i}}=1 \text { for all } x \in W
$$

where $m(W)>0$. Thus, if there exists a weakly wandering set of positive measure, we have a contradiction to (1).

Conversely, suppose condition (1) is not satisfied. Then there exist a measurable function $f(x)>0$ a.e., a sequence of integers $\left\{n_{i} \mid i=1,2, \cdots\right\}$, and a measurable set $A$ with $m(A)>0$ such that

$$
\sum_{i=1}^{\infty} f\left(\phi^{n_{i}} x\right)<\infty \quad \text { for all } x \in A .
$$

In case $m(X)=\infty$ but $\sigma$-finite, we have $X=\bigcup_{i=1}^{\infty} B_{i}$ where $B_{i} \cap B_{j}=\varnothing$ for $i \neq j$ and $0<m\left(B_{i}\right)<\infty$ for $i=1,2, \cdots$. We introduce the measure $m^{\prime}$ defined on $\mathscr{B}$ as follows:

$$
m^{\prime}(A)=\sum_{i=1}^{\infty} \frac{1}{2^{i}} \frac{m\left(A \cap B_{i}\right)}{m\left(B_{i}\right)} \text { for all } A \in \mathscr{B} .
$$

It follows that $m^{\prime}(X)=1$ and $m^{\prime}$ is equivalent to $m$.

Next, we let $\varepsilon>0$ be an arbitrary positive number. Since $m^{\prime}(X)<\infty$ and $f(x)>0$ a.e., it follows that there exist a positive number $\delta_{1}>0$ and a measurable set $C_{1}$ with $m^{\prime}\left(C_{1}\right)<\varepsilon$ such that $f(x) \geqq \delta_{1}>0$ for all $x \in X-C_{1}$. Using (2) we obtain

$$
\sum_{i=1}^{\infty} \chi_{X-c_{1}}\left(\phi^{n_{i}} x\right) \leqq \frac{1}{\delta_{1}} \sum_{i=1}^{\infty} f\left(\phi^{n_{i}} x\right)<\infty \text { for all } x \in A
$$

where $\chi_{X-c_{1}}(x)$ is the characteristic function of the set $X-C_{1}$. This means that for all $x \in A$ there exists a positive integer $k=k(x)$ such that $\phi^{n_{i}} x \in C_{1}$ for all $i \geqq k=k(x)$. We let 


$$
A(k)=\{x \in A \mid k(x)=k\} \text { for } k=1,2, \cdots .
$$

It follows then that for some positive number $\eta>0$ with $0<\eta<a=m^{\prime}(A)$, there exists an index $k_{1}$ such that $A_{1}=A\left(k_{1}\right) \subset A$ and $m^{\prime}\left(A-A_{1}\right)<\eta / 2$. Since $\phi^{n_{i}} x \in C_{1}$ for all $x \in A_{1}$ and all $i \geqq k_{1}$, we conclude that

$$
\lim _{i \rightarrow \infty} \inf ^{\prime}\left(\phi^{n_{i}} A_{1}\right) \leqq m^{\prime}\left(C_{1}\right) \leqq \varepsilon .
$$

Next, for each $p=2,3, \cdots$ we perform inductively the same construction as above with $\varepsilon / p$ replacing $\varepsilon$. Thus, for each $p=2,3, \cdots$ we obtain a positive number $\delta_{p}>0$ and a measurable set $C_{p}$ with $m^{\prime}\left(C_{p}\right) \leqq \varepsilon / p$ such that $f(x) \geqq \delta_{p}>0$ for all $x \in X-C_{p}$. Consequently, we construct the set $A_{p}$ contained in the previously constructed set $A_{p-1}$ with the property that

and

$$
m^{\prime}\left(A_{p-1}-A_{p}\right)<\eta / 2^{p}
$$

$$
\underset{i \rightarrow \infty}{\lim \inf } m^{\prime}\left(\phi^{n_{i}} A_{p}\right) \leqq m^{\prime}\left(C_{p}\right) \leqq \varepsilon / p
$$

Now let $A^{\prime}=\bigcap_{p=1}^{\infty} A_{p}$. It follows that

$$
\begin{aligned}
\liminf _{n \rightarrow \infty} m^{\prime}\left(\phi^{n} A^{\prime}\right) & \leqq \liminf _{i \rightarrow \infty} m^{\prime}\left(\phi^{n_{i}} A^{\prime}\right) \leqq \liminf _{i \rightarrow \infty} m^{\prime}\left(\phi^{n_{i}} A_{p}\right) \\
& \leqq m^{\prime}\left(C_{p}\right) \leqq \varepsilon / p \rightarrow 0 \quad \text { as } p \rightarrow \infty
\end{aligned}
$$

Furthermore,

$$
\begin{aligned}
m^{\prime}\left(A^{\prime}\right) & =m^{\prime}\left(\bigcap_{p=1}^{\infty} A_{p}\right)=m^{\prime}\left(A-\bigcup_{p=1}^{\infty}\left(A_{p-1}-A_{p}\right)\right) \\
& \geqq m^{\prime}(A)-\sum_{p=1}^{\infty} \eta / 2^{p}=a-\eta>0 .
\end{aligned}
$$

Thus, we obtain a measurable set $A^{\prime}$ with $m^{\prime}\left(A^{\prime}\right)>0$ and $\lim \inf _{n \rightarrow \infty} m^{\prime}\left(\phi^{n} A^{\prime}\right)=0$. Using Lemma 4 of [1] we can construct a weakly wandering set of positive measure $\left(m^{\prime}\right)$. Since $m$ and $m^{\prime}$ are equivalent, this completes the proof of the theorem.

Let us call a collection $\left\{A_{i} \mid i=1,2, \cdots\right\}$ of measurable sets a decomposition of the space $X$ if the following two conditions are satisfied:

i. $A_{i} \cap A_{j}=\varnothing$ if $i \neq j$,

ii. $\bigcup_{i=1}^{\infty} A_{i}=X$.

THEOREM 2. Let $\phi$ be a measurable and nonsingular transformation defined on a $\sigma$-finite measure space $(X, \mathscr{B}, m)$. Then there does not exist a weakly wandering set of positive measure if and only if 
for any sequence of integers $\left\{n_{i} \mid i=1,2, \cdots\right\}$, for any decomposition $\left\{A_{k} \mid k=1,2, \cdots\right\}$ of the space $X$, and for almost all $x \in X$ there exists some $k=k(x)$ such that $\phi^{n_{i}} x \in A_{k}$ for infinitely many $i$.

Proof. Let $W$ be a set of positive measure and weakly wandering under the sequence $\left\{n_{i} \mid i=1,2, \cdots\right\}$. Let us put

and

$$
A_{1}=X-\bigcup_{i=1}^{\infty} \phi^{n_{i}} W
$$

$$
A_{k}=\phi^{n_{k-1}} W \quad \text { for } k=2,3, \cdots .
$$

It follows then that $\left\{A_{k} \mid k=1,2, \cdots\right\}$ is a decomposition of the space $X$ and, furthermore, for the above sequence $\left\{n_{i}\right\}$, for every $k=1,2, \cdots$, and for all $x \in W$ we have $\phi^{n_{i}} x \in A_{k}$ for at most one $i=i(k)$. Since $m(W)>0$ this is a contradiction to (4).

Conversely, suppose condition (4) is not satisfied. Then there exist a sequence of integers $\left\{n_{i} \mid i=1,2, \cdots\right\}$, a decomposition $\left\{A_{k} \mid k=1,2, \cdots\right\}$ of the space $X$, and a measurable set $A$ with $m(A)>0$ such that for all points $x \in A$ and for every $k=1,2, \cdots$, we have $\phi^{n_{i}} x \in A_{k}$ for at most finitely many $i$. Let $m(A)=a>0$. Then, for any $\varepsilon$ with $0<\varepsilon<a$, and for each $k=1,2, \cdots$, we can find a subset $B_{k}$ of $A$ with $m\left(B_{k}\right)<\varepsilon / 2^{k}$, and some positive integer $M_{k}$ such that $\phi^{n_{i}} x \in A_{k}$ for at most $M_{k}$ times for all $x \in A-B_{k}$. Let

Then

$$
A^{\prime}=A-\bigcup_{k=1}^{\infty} B_{k} .
$$

$$
m\left(A^{\prime}\right) \geqq m(A)-\sum_{k=1}^{\infty} m\left(B_{k}\right) \geqq a-\varepsilon>0 .
$$

We now define the real-valued function $f(x)$ on $X$ as follows:

$$
f(x)=\sum_{k=1}^{\infty} \frac{1}{2^{k} M_{k}} \chi_{A_{k}}(x),
$$

where $\chi_{A_{k}}(x)$ is the characteristic function of the set $A_{k}$ for $k=1,2, \cdots$. It follows that $f(x)$ is a measurable function defined on $X$ with $f(x)>0$ a.e.; furthermore, for the above sequence $\left\{n_{i} \mid i=1,2, \cdots\right\}$,

$$
\sum_{i=1}^{\infty} f\left(\phi^{n_{i}} x\right)<\infty \text { for all } x \in A^{\prime} .
$$

Since $m\left(A^{\prime}\right)>0$ this contradicts (1). By making use of Theorem 1 we complete the proof. 
We say that a measure $\mu$ defined on $(X, \mathscr{B})$ is invariant under the measurable transformation $\phi$ if $\mu(B)=\mu(\phi B)=\mu\left(\phi^{-1} B\right)$ for every measurable set $B$.

COROLlary. Both condition (1) and condition (4) on a measurable and nonsingular transformation $\phi$ defined on a $\sigma$-finite measure space $(X, \mathscr{B}, m)$ are necessary and sufficient conditions for the existence of a finite, invariant, and equivalent measure $\mu$.

Proof. Follows from Theorem 1 and Theorem 2 above and Theorem 1 of [1] where it was shown that for a given measurable and nonsingular transformation $\phi$ defined on a measure space $(X, \mathscr{B}, m)$ there exists a finite, invariant, and equivalent measure $\mu$ if and only if there does not exist any weakly wandering set of positive measure.

3. Ergodic measure-preserving transformations defined on an infinite measure space. In this section we consider an infinite but $\sigma$-finite measure space $(X, \mathscr{B}, m)$. For example, the real line with the usual Lebesgue measurable subsets and the Lebesgue measure $m$ defined on it with the measure of the whole space infinite. A measurable transformation $\phi$ defined on $(X, \mathscr{B}, m)$ is said to be ergodic if $\phi B=B$ implies $m(B)=0$ or $m(X-B)=0$ for any measurable set $B$. The transformation $\phi$ is said to be measure-preserving if the measure $m$ is invariant under $\phi$. A sequence of integers $\left\{n_{i} \mid i=1,2, \cdots\right\}$ is said to be a weakly wandering sequence for the transformation $\phi$ if there exists a measurable set $W$ of positive measure which is weakly wandering under the sequence $\left\{n_{i} \mid i=1,2, \cdots\right\}$.

It is easy to see that an ergodic measure-preserving transformation $\phi$ defined on an infinite measure space $(X, \mathscr{B}, m)$ does not admit any finite invariant measure $\mu$ equivalent to $m$, see [1]. In this section we study the relationship of such a transformation to condition (1) and condition (4) in more detail. It was shown in [1] (see Theorem 2 of [1] and also Theorem 1 of [2]) that every ergodic measurepreserving transformation $\phi$ defined on an infinite measure space $(X, \mathscr{B}, m)$ possesses weakly wandering sets of positive measure. Making use of this fact, we prove the following:

THEOREM 3. Let $\phi$ be an ergodic measure-preserving transformation defined on an infinite measure space $(X, \mathscr{B}, m)$, and let $\left\{n_{i} \mid i=1,2, \cdots\right\}$ be a weakly wandering sequence. Then for any integrable function $f(x)$ defined on $X$

$$
\sum_{i=1}^{\infty}\left|f\left(\phi^{n_{i}} x\right)\right|<\infty \quad \text { a.e. }
$$

Proof. Let $W$ be a set of positive measure and weakly wandering under the sequence $\left\{n_{i} \mid i=1,2, \cdots\right\}$, and let $f(x)$ be an integrable function. It follows then that 
(5)

$$
\begin{aligned}
\infty & >\int_{X}|f(x)| d m(x) \geqq \int_{\bigcup_{r=1 \phi^{n_{i} W}}^{\infty}}|f(x)| d m(x) \\
& =\sum_{i=1}^{\infty} \int_{\phi^{n_{i} W}}|f(x)| d m(x) .
\end{aligned}
$$

In each summand of the last member of the above inequality we perform a change of variable of integration, and since the measure $m$ is invariant under the transformation $\phi$ it follows that

$$
\sum_{i=1}^{\infty} \int_{\phi^{n_{i}} W}|f(x)| d m(x)=\sum_{i=1}^{\infty} \int_{W}\left|f\left(\phi^{n_{i}} x\right)\right| d m(x) .
$$

By the Monotone Convergence Theorem, the last member of equation (6) is equal to

$$
\int_{W} \sum_{i=1}^{\infty}\left|f\left(\phi^{n_{i}} x\right)\right| d m(x)
$$

Combining (5), (6), and (7) we conclude that

$$
\sum_{i=1}^{\infty}\left|f\left(\phi^{n_{i}} x\right)\right|<\infty \text { for almost all } x \in W .
$$

Since $\phi$ is ergodic and $W$ is a set of positive measure, it follows that

$$
m\left(X-\bigcup_{n=-\infty}^{\infty} \phi^{n} W\right)=0 .
$$

It is easy to see that if $W$ is a weakly wandering set under the sequence $\left\{n_{i} \mid i=1,2, \cdots\right\}$, then for any integer $n=0, \pm 1, \pm 2, \cdots$ the set $\phi^{n} W$ is again weakly wandering under the same sequence $\left\{n_{i} \mid i=1,2, \cdots\right\}$. Next, for every integer $n=0, \pm 1, \pm 2, \cdots$ we repeat the above argument with the set $\phi^{n} W$ replacing the set $W$ and conclude that

$$
\sum_{i=1}^{\infty} f\left(\phi^{n_{i}} x\right)<\infty \text { for almost all } x \in \phi^{n} W .
$$

Combining (9) and (10) we conclude the proof of the theorem.

COROLlARY 1. Let $\phi$ be an ergodic measure-preserving transformation defined on an infinite measure space $(X, \mathscr{B}, m)$ and let $\left\{n_{i} \mid i=1,2, \cdots\right\}$ be a weakly wandering sequence. Then for any measurable set $A$ with $m(A)<\infty$ and for almost all $x \in X$ we have $\phi^{n_{i}} x \in A$ for at most finitely many $i$.

Proof. We note that the characteristic function $\chi_{A}(x)$ of the set $A$ is an integrable function. The rest of the proof is an immediate consequence of Theorem 3. 
COROLlaRY 2. Let $\phi$ be an ergodic measure-preserving transformation defined on an infinite measure space $(X, \mathscr{B}, m)$ and let $\left\{n_{i} \mid i=1,2, \cdots\right\}$ be a weakly wandering sequence. Let $\left\{A_{k} \mid k=1,2, \cdots\right\}$ be a decomposition of the space $X$ with $m\left(A_{k}\right)<\infty$ for $k=1,2, \cdots$. Then for every $k=1,2, \cdots$ and for almost all $x \in X$ we have $\phi^{n_{i}} x \in A_{k}$ for at most finitely many $i$.

Proof. That there are such decompositions follows from the definition of $\sigma$-finiteness of the measure space $(X, \mathscr{B}, m)$. The proof then is an immediate consequence of Corollary 1.

4. Markov processes. Since we can always replace the measure $m$ by a measure which is totally finite and is equivalent to $m$, see (3), we shall assume that $m(x)<\infty$.

Let us denote by $P(x, B)$ the transition probability of a temporally homogeneous, discrete time Markov process given in $(X, \mathscr{B})$. By this we mean that $P(x, B)$ satisfies the following conditions:

(i) $P(x, B)$ is a real-valued function defined for every pair $(x, B)$ where $x \in X$ and $B \in \mathscr{B}$, and $0 \leqq P(x, B) \leqq 1$.

(ii) For every fixed $x, P(x, B)$ is a measure defined on $(X, \mathscr{B})$ with $P(x, X)=1$.

(iii) For every fixed $B \in \mathscr{B}, P(x, B)$ is a measurable function.

We define the $n$th transition probability of the process recurrently by:

and

$$
P^{1}(x, B)=P(x, B)
$$

$$
P^{n}(x, B)=\int_{X} P^{n-1}(y, B) P(x, d y) \text { for } n=2,3, \cdots .
$$

Then it is clear that for each positive integer $n=1,2, \cdots$, the $n$th transition probability satisfies the same conditions (i), (ii) and (iii) above. The $n$th transition probability $P^{n}(x, B)$ represents the probability that a "path" of the process starting from the point $x$ will go into the set $B$ in $n$ steps. In this note we shall have no occasion to deal with the probability model ("paths" of the process), but shall treat everything in terms of transition probabilities. Therefore, in the sequel, we shall abuse the terminology and say a Markov process to mean its transition probability $P(x, B)$. Let us call the process $P(x, B)$ nonsingular with respect to the measure $m$ if $m(B)=0$ implies that $P(x, B)=0$ a.e. $(m)$. It is easy to verify that if $P(x, B)$ is nonsingular, then so is $P^{n}(x, B)$ for each $n=1,2, \cdots$. In what follows we shall always assume the nonsingularity of the process $P(x, B)$ with respect to the given measure $m$.

Suppose $\phi$ is a (not necessarily one-to-one) measurable and nonsingular transformation defined on $(X, \mathscr{B}, m)$. For such a transformation $\phi$ to be measurable we mean $\phi^{-1} B$ is measurable for every measurable set $B$, and to be nonsingular we mean $m\left(\phi^{-1} B\right)=0$ for every measurable set $B$ with $m(B)=0$. Now given such a transformation $\phi$, it can be regarded as a special type of Markov process whose transition probability is given by the formula: 


$$
P(x, B)=\chi_{\phi^{-1}(B)}(x)=\chi_{B}(\phi x),
$$

where $\chi_{\phi^{-1}(B)}(x)$ and $\chi_{B}(x)$ denote the characteristic functions of the sets $\phi^{-1}(B)$ and $B$, respectively.

We say that a measure $\mu$ defined on the measurable space $(X, \mathscr{B})$ is invariant for the process $P(x, B)$ if for every $B \in \mathscr{B}$

$$
\mu(B)=\int_{X} P(x, B) d \mu(x) .
$$

If $P(x, B)$ is generated by a point transformation $\phi$ as indicated by (11), then $\mu$ is an invariant measure for the process $P(x, B)$ if and only if $\phi$ is a measurepreserving transformation with respect to the measure $\mu$; i.e., $\mu\left(\phi^{-1} B\right)=\mu(B)$ for every measurable set $B$.

Let us denote by $L^{1}(m)$ the Banach space of all real-valued, measurable, and integrable functions on $X$, and by $L^{\infty}(m)$ the Banach space of all real-valued, measurable, and essentially bounded functions on $X$. Norms in these spaces are defined as usual. Two functions in $L^{1}(m)$ or $L^{\infty}(m)$ which differ only on a set of measure zero will be identified.

A nonsingular Markov process $P(x, B)$ gives rise to a linear operator $T$ of $L^{1}(m)$ into itself and a linear operator $U$ of $L^{\infty}(m)$ into itself by the following formulae:

$$
\begin{aligned}
T: f \rightarrow T f & =\frac{d}{d m}\left[\int_{X} f(x) P(x, \cdot) d m(x)\right], \\
U: g \rightarrow U g & =\int_{X} g(y) P(x, d y),
\end{aligned}
$$

where $d / d m$ denotes the Radon-Nikodym derivative with respect to the measure $m$. It is easy to check that both $T$ and $U$ are positive operators, that $\|T\|_{1}=1,\|U\|_{\infty}=1$ and that $T$ and $U$ are adjoint operators of each other; i.e., for every pair of functions $f, g$ with $f \in L^{1}(m), g \in L^{\infty}(m)$,

$$
\int_{X} f(x) U g(x) d m(x)=\int_{X} T f(x) g(x) d m(x) .
$$

It was shown by E. Hopf [3] that with respect to the operator $T$ one can always decompose the space $X$ into two disjoint parts; the conservative part $C$ and the dissipative part $D$, which are characterized by the following properties: for every strictly positive function $f \in L^{1}(m)$,

$$
\sum_{n=0}^{\infty} T^{n} f(x)=\infty \text { for almost all } x \in C
$$

and for every function $h \in L^{1}(m)$,

$$
\left|\sum_{n=0}^{\infty} T^{n} h(x)\right|<\infty \text { for almost all } x \in D .
$$


It is not difficult to show that if the Markov process $P(x, B)$ possesses a finite invariant measure $\mu$ equivalent to $m$, then the dissipative part $D$ must be a set of measure zero. However, the condition $X=C$ is not sufficient for the existence of a finite, invariant, and equivalent measure $\mu$ for the process $P(x, B)$. In fact, we can state the following:

THEOREM 4. If there exists a finite, invariant, and equivalent measure for the process $P(x, B)$, then

$$
\text { for any measurable function } f(x) \text { with } f(x)>0 \text { a.e., and for }
$$
any sequence of positive integers $\left\{n_{i} \mid i=1,2, \cdots\right\}$.

$$
\sum_{i=1}^{\infty} T^{n_{i}} f(x)=\infty \text { a.e. }
$$

Conversely, if there exists a function $f(x)$ in $L^{1}(m)$ with $f(x)>0$ a.e. and which has the property that

$$
\sum_{i=1}^{\infty} T^{n_{i}} f(x)=\infty \text { a.e. }
$$

for any sequence of positive integers $\left\{n_{i} \mid i=1,2, \cdots\right\}$, then there exists a finite, invariant, and equivalent measure for the process $P(x, B)$.

Proof. In order to prove the first part of the theorem, let us suppose that there exist a measurable function $f(x)$ with $f(x)>0$ a.e., an infinite sequence of positive integers $\left\{n_{i} \mid i=1,2, \cdots\right\}$, and a set $A \in \mathscr{B}$ with $m(A)>0$ such that

$$
\sum_{i=1}^{\infty} T^{n_{i}} f(x)<\infty \text { for all } x \in A
$$

We may suppose that $f(x)$ belongs to $L^{1}(m)$. We define a new measure $\mu$ on $(X, \mathscr{B})$ by:

$$
\mu(B)=\int_{B} f(x) d m(x) \text { for all } B \in \mathscr{B} .
$$

It is clear that $\mu$ is totally finite and is equivalent to $m$. From (13) it follows that

$$
\lim _{i \rightarrow \infty} T^{n_{i}} f(x)=0 \text { for all } x \in A \text {. }
$$

By Egorov's theorem, we can find a measurable subset $A^{\prime}$ of $A$ and some constant $M>0$ such that $m\left(A^{\prime}\right)>0$ and for all $x \in A^{\prime}$

$$
\left|T^{n_{i}} f(x)\right| \leqq M \text { for all } i=1,2, \cdots \text {. }
$$

Using the Dominated Convergence Theorem, it follows that

Consequently,

$$
\lim _{i \rightarrow \infty} \int_{A^{\prime}} T^{n_{i}} f(x) d m(x)=0 .
$$




$$
\lim _{i \rightarrow \infty} \int_{X} U^{n_{i}} \chi_{A^{\prime}}(x) f(x) d m(x)=\lim _{i \rightarrow \infty} \int_{X} P^{n_{i}}\left(x, A^{\prime}\right) d \mu(x)=0
$$

This implies that

$$
\liminf _{n \rightarrow \infty} \int_{X} P^{n}\left(x, A^{\prime}\right) d \mu(x)=0
$$

where $\mu\left(A^{\prime}\right)>0$ by the equivalence of $\mu$ and $m$. By Theorem 3 of $[4], P(x, B)$ cannot have a finite invariant measure which is equivalent to $\mu$ (hence to $m$ ). This proves the first part of the theorem.

To prove the second part of the theorem, let $f(x)$ be the function satisfying the properties stated in the hypothesis. Let us denote again

$$
\mu(B)=\int_{B} f(x) d m(x),
$$

and consider the Banach space $L^{1}(\mu)$. It is obvious that a measurable function $h(x)$ belongs to $L^{1}(\mu)$ if and only if $h(x) f(x)$ belongs to $L^{1}(m)$. We define a linear operator $S$ of $L^{1}(\mu)$ into itself by

$$
S: h \rightarrow S h=\frac{d}{d \mu}\left[\int_{X} h(x) P(x, \cdot) d \mu(x)\right] .
$$

It is clear that $S$ and $U$ are adjoint operators of each other, whereby $U$ is regarded as a linear operator on $L^{\infty}(\mu)$ (which is actually identical with $L^{\infty}(m)$ ).

It is easy to verify that the operators $T$ and $S$ are related by the equation:

$$
T^{k}(h f)=f S^{k} h \text { for each } k=1,2, \cdots .
$$

Clearly, the function $1(x)$ which takes constant value one a.e. $(m)$ (hence a.e. $(\mu)$ ) belongs to $L^{1}(\mu)$; thus, by taking $h$ to be 1 , we get

$$
f S^{k} \mathbf{1}=T^{k} f \text { for each } k=1,2, \cdots \text {. }
$$

Therefore, our hypothesis implies that, for any sequence of positive integers $\left\{n_{i} \mid i=1,2, \cdots\right\}$,

$$
\left.f(x) \sum_{i=1}^{\infty} S^{n_{i}} \mathbf{1}(x)=\infty \text { a.e. }(m) \text { (hence a.e. }(\mu)\right) .
$$

Since $f(x)$ belongs to $L^{1}(m)$, it follows that $0<f(x)<\infty$ a.e. $(m)$ (hence a.e. $(\mu)$ ). Consequently, for any sequence of positive integers $\left\{n_{i} \mid i=1,2, \cdots\right\}$,

$$
\sum_{i=1}^{\infty} S^{n_{i}} \mathbf{1}(x)=\infty \text { a.e. }(\mu)
$$

Now suppose that for some measurable set $E$ and some sequence of positive integers $\left\{n_{j} \mid j=1,2, \cdots\right\}$ 


$$
\sum_{j=1}^{\infty} \int_{X} P^{n_{j}}(x, E) d \mu(x)<\infty
$$

Then, since

$$
\sum_{j=1}^{\infty} \int_{X} P^{n_{j}}(x, E) d \mu(x)=\sum_{j=1}^{\infty} \int_{E} S^{n_{j}} \mathbf{1}(x) d \mu(x)=\int_{E} \sum_{j=1}^{\infty} S^{n_{j}} \mathbf{1}(x) d \mu(x),
$$

we must have $\mu(E)=0$. Therefore, by Theorem 4 of [4] there exists a finite measure $v$ which is equivalent to $\mu$ (hence to $m$ ) and is invariant for $P(x, B)$. This completes the proof of the theorem.

Corollary. Suppose there exists one function $f(x)$ in $L^{1}(m)$ with $f(x)>0$ a.e. and which has the property that, for any sequence of positive integers $\left\{n_{i} \mid i=1,2, \cdots\right\}$,

$$
\sum_{i=1}^{\infty} T^{n_{i}} f(x)=\infty \text { a.e. }
$$

Then for any measurable function $g(x)$ with $g(x)>0$ a.e. and for any sequence of positive integers $\left\{n_{i} \mid i=1,2, \cdots\right\}$, we have

$$
\sum_{i=1}^{\infty} T^{n_{i}} g(x)=\infty \text { a.e. }
$$

Proof. Follows directly from Theorem 4.

We note that it is possible to construct a Markov process $P(x, B)$ for which $X=C$ but

$$
\sum_{i=1}^{\infty} T^{n_{i}} f(x)<\infty \text { a.e. }
$$

for some measurable function $f(x)$ with $f(x)>0$ a.e. and some sequence of positive integers $\left\{n_{i} \mid i=1,2, \cdots\right\}$.

The assertions in Theorem 4 were stated in terms of the operator $T$. It is interesting to know whether similar assertions stated in terms of the adjoint operator $U$ would be valid. It turns out that an analogue of the first assertion of Theorem 4 is valid with respect to the operator $U$, while the second assertion is not. Namely,

THEOREM 5. If there exists a finite, invariant, and equivalent measure $v$ for the Markov process $P(x, B)$, then

for any measurable function $f(x)$ with $f(x)>0$ a.e., and for any sequence of positive integers $\left\{n_{i} \mid i=1,2, \cdots\right\}$, we have

$$
\sum_{i=1}^{\infty} U^{n_{i}} f(x)=\infty \text { a.e. }
$$


However, there exists a Markov process $P(x, B)$ for which condition (14) is satisfied and yet there does not exist a finite, invariant, and equivalent measure.

Proof. In order to prove the first part of the theorem, let us define an operator $R$ on $L^{1}(v)$ into itself by

$$
R: h \rightarrow R h=\frac{d}{d v}\left[\int_{X} h(x) P(x, \cdot) d v(x)\right] .
$$

Then clearly, $R$ and $U$ are adjoint operators of each other, whereby we regard $U$ as an operator on $L^{\infty}(v)$ into itself. Now let us suppose that condition (14) is not satisfied. Then, there exist a measurable function $f(x)$ with $f(x)>0$ a.e., a sequence of positive integers $\left\{n_{i} \mid i=1,2, \cdots\right\}$, and a measurable set $A$ with $m(A)>0$ such that

$$
\sum_{i=1}^{\infty} U^{n_{i}} f(x)<\infty \quad \text { for all } x \in A .
$$

We may assume without loss of generality that $f(x)$ belongs to $L^{\infty}(v)$. From $(15)$ it follows that

$$
\lim _{i \rightarrow \infty} U^{n_{i}} f(x)=0 \text { for all } x \in A .
$$

Since $\left|U^{n_{i}} f(x)\right| \leqq v$-ess. sup $|f(x)|$ for all $i$, and since $v$ is a finite measure, we conclude that

$$
\lim _{i \rightarrow \infty} \int_{A} U^{n_{i}} f(x) d v(x)=0
$$

which implies that

$$
\lim _{i \rightarrow \infty} \int_{X} f(x) R^{n_{i}} \chi_{A}(x) d v(x)=0 .
$$

Thus, we can find a subsequence $\left\{n_{j}\right\}$ of the sequence $\left\{n_{i}\right\}$ such that

$$
R^{n_{j}} \chi_{A}(x) \rightarrow 0 \text { a.e. as } j \rightarrow \infty \text {. }
$$

The invariance of the measure $v$ implies that

$$
R^{k} 1=1 \text { a.e. for each } k=1,2, \cdots,
$$

so that, by the positivity of $R$,

$$
R^{k} \chi_{A}(x) \leqq 1 \text { a.e. for each } k=1,2, \cdots \text {. }
$$

Thus, the sequence $\left\{R^{n_{j}} \chi_{A}(x)\right\}$ tends to 0 a.e. boundedly as $j \rightarrow \infty$, and this implies that

$$
\lim _{j \rightarrow \infty} \int_{X} R^{n_{j}} \chi_{A}(x) d \nu(x)=0
$$


But for each $j=1,2, \cdots$,

$$
\int_{X} R^{n_{j}} \chi_{A}(x) d v(x)=\int_{A} U^{n_{j}} \mathbf{1}(x) d v(x)=v(A)
$$

so that we must have $v(A)=0$. Since $v$ is equivalent to $m$, we have a contradiction.

In order to show the second part of the theorem, let us take the measure space $(X, \mathscr{B}, m)$, where $X$ is the unit interval $[0,1]$ with the $\sigma$-field $\mathscr{B}$ of Lebesgue measurable subsets of $X$ and the ordinary Lebesgue measure $m$ defined on it with $m(X)=1$. We consider on this measure space a point transformation $\phi$ defined as follows:

$$
\phi(x)= \begin{cases}2 x & \text { if } x \in[0,1 / 2) \\ x & \text { if } x \in[1 / 2,1]\end{cases}
$$

It is clear that $\phi$ is a many-to-one, measurable, and nonsingular transformation. We consider now a Markov process $P(x, B)$ generated by this transformation $\phi$, where $P(x, B)$ is given by (11). Then, the linear operator $U$ associated with this process is given by:

$$
U: f \rightarrow U f(x)=\int_{X} f(y) P(x, d y)=f(\phi x) .
$$

It is clear from the definition of the transformation $\phi$ that this Markov process satisfies the condition (14). On the other hand, it is also easy to see that any invariant measure $\mu$ for the process $P(x, B)$ must have the property that $\mu[0,1 / 2)=0$. Therefore, this process does not have any finite, invariant, and equivalent measure. This completes the proof of the theorem.

In the next theorem we consider the analogue of condition (4) for a Markov process.

THEOREM 6. There exists a finite, invariant, and equivalent measure $\mu$ for the Markov process $P(x, B)$ if and only if

for any decomposition $\left\{A_{k} \mid k=1,2, \cdots\right\}$ of the space $X$,

for any measurable set $B$ with $m(B)>0$, and for any sequence

of positive integers $\left\{n_{i} \mid i=1,2, \cdots\right\}$, there exists some $k$

(depending on the set $B$ and the sequence $\left\{n_{i}\right\}$ ) such that

$$
\sum_{i=1}^{\infty} \int_{A_{k}} P^{n_{i}}(x, B) d m(x)=\infty
$$

Proof. To prove the sufficiency, let us take a decomposition of the space $X$ which consists of $X$ alone. Condition (17) states that for any measurable set $B$ with $m(B)>0$ and for any sequence of positive integers $\left\{n_{i} \mid i=1,2, \cdots\right\}$,

$$
\sum_{i=1}^{\infty} \int_{X} P^{n_{i}}(x, B) d m(x)=\infty \text {. }
$$


Therefore, for any measurable set $B$ with $m(B)>0$,

$$
\underset{n \rightarrow \infty}{\liminf } \frac{1}{n} \sum_{k=0}^{n-1} \int_{X} P^{k}(x, B) d m(x)>0 .
$$

By Theorem 3 of [4], there exists a finite, invariant, and equivalent measure $\mu$ for the Markov process $P(x, B)$.

To prove the necessity, let us suppose that the Markov process $P(x, B)$ has a finite, invariant, and equivalent measure $\mu$ and that there exist some decomposition $\left\{A_{k} \mid k=1,2, \cdots\right\}$ of the space $X$, a measurable set $B$ with $m(B)>0$, and a sequence of positive integers $\left\{n_{i} \mid i=1,2, \cdots\right\}$ such that

$$
\sum_{i=1}^{\infty} \int_{A_{k}} P^{n_{1}}(x, B) d m(x)<\infty \text { for all } k=1,2, \cdots
$$

Let us put

$$
M_{k}=\max \left\{1, \sum_{i=1}^{\infty} \int_{A_{k}} P^{n_{i}}(x, B) d m(x)\right\} \text { for } k=1,2, \cdots,
$$

and define

$$
f(x)=\sum_{k=1}^{\infty} \frac{1}{2^{k} M_{k}} \chi_{A_{k}}(x) .
$$

Then $f(x)$ is a measurable function on $X$ with $f(x)>0$ a.e., and $f(x)$ belongs to $L^{\infty}(m)$ (and, therefore, to $L^{1}(m)$ ). It is easy to show that for each $j=1,2, \ldots$

$$
T^{j} f(x)=\sum_{k=1}^{\infty} \frac{1}{2^{k} M_{k}} T^{j} \chi_{A_{k}}(x)
$$

Now,

$$
\begin{aligned}
\sum_{i=1}^{\infty} \int_{B} T^{n_{i}} f(x) d m(x) & =\sum_{i=1}^{\infty} \int_{B} \sum_{k=1}^{\infty} \frac{1}{2^{k} M_{k}} T^{n_{i}} \chi_{A_{k}}(x) d m(x) \\
& =\sum_{i=1}^{\infty} \sum_{k=1}^{\infty} \frac{1}{2^{k} M_{k}} \int_{B} T^{n_{i}} \chi_{A_{k}}(x) d m(x) \\
& =\sum_{k=1}^{\infty} \frac{1}{2^{k} M_{k}} \sum_{i=1}^{\infty} \int_{A_{k}} P^{n_{t}}(x, B) d m(x)
\end{aligned}
$$

$\leqq 1$.

By the Monotone Convergence Theorem it follows that

$$
\int_{B} \sum_{i=1}^{\infty} T^{n_{i}} f(x) d m(x)=\sum_{i=1}^{\infty} \int_{B} T^{n_{i}} f(x) d m(x),
$$

from which it follows that 


$$
\sum_{i=1}^{\infty} T^{n_{i}} f(x)<\infty \text { for almost all } x \in B
$$

Thus we have a contradiction to the first part of Theorem 4, and this completes the proof.

Suppose in condition (17) of Theorem 6 we interchange the role of $A_{k}$ and $B$, i.e., suppose we consider the following condition: For any decomposition $\left\{A_{k} \mid k=1,2, \cdots\right\}$ of the space $X$, for any measurable set $B$ with $m(B)>0$, and for any sequence of positive integers $\left\{n_{i} \mid i=1,2, \cdots\right\}$, there exists an index $k$ (depending on the set $B$ and the sequence $\left\{n_{i}\right\}$ ) such that

$$
\sum_{i=1}^{\infty} \int_{B} P^{n_{i}}\left(x, A_{k}\right) d m(x)=\infty .
$$

Then, the necessity of this condition for the existence of a finite, invariant, and equivalent measure for the process $P(x, B)$ can be proved in exactly the same manner as the necessity part of Theorem 6 . However, this condition is not sufficient, as the same example mentioned in the proof of Theorem 5 satisfies this condition.

\section{BIBLIOGRAPHY}

1. A. B. Hajian and S. Kakutani, Weakly wandering sets and invariant measures, Trans. Amer. Math. Soc. 110 (1964), 136-151.

2. A. B. Hajian, On ergodic measure preserving transformations defined on an infinite measure space, Proc. Amer. Math. Soc. 16 (1965), 45-48.

3. E. Hopf, The general temporally discrete Markov process, J. Rational Mech. Anal. 3 (1954), $13-45$.

4. Y. Ito, Invariant measures for Markov processes, Trans. Amer. Math. Soc. 110 (1964), 152-184.

CORNELL UNIVERSTTY, ItHACA, NeW York

BROWN UNIVERSITY, Providence, Rhode IsLand 